\title{
Envelhecimento e força muscular - breve revisão
}

\author{
Joana Carvalho \\ José MC Soares
}

https://doi.org/10.5628/rpcd.04.03.79

\author{
Faculdade de Ciências de Desporto e de Educação Física \\ Universidade do Porto \\ Portugal
}

\section{RESUMO}

O envelhecimento é um dos fenómenos que mais se evidencia nas sociedades actuais. A preocupação com o bem-estar e a qualidade de vida desta população, por parte de diferentes entidades, é cada vez mais notório. A capacidade de realizar diferentes actividades diárias, actividades laborais ou recreacionais é determinada, em grande parte, pela capacidade de desenvolver força muscular $(15,52)$. A diminuição da força que ocorre com o envelhecimento é um dos fenómenos mais amplamente estudados no que se refere às alterações fisiológicas induzidas pela idade (98). Para além da observação da diminuição da força muscular com a idade, a investigação tem também vindo a abordar os possíveis efeitos da actividade física nesta capacidade. Numerosos estudos têm referido a efectividade de diferentes tipos de exercício físico na manutenção e/ou melhoria da força e resistência musculares em idosos (para refs. ver 5). Todavia, o exercício físico só é benéfico quando orientado segundo determinadas regras e princípios. Uma actividade irregular, nomeadamente o treino de força, desorganizada, sem nenhuma orientação profissional pode ser prejudicial, particularmente no que respeita ao sistema cardiovascular e locomotor passivo. Neste sentido, o objectivo deste trabalho é rever a literatura sobre o envelhecimento muscular, a sua influência na qualidade de vida e a efectividade dos programas de actividade física na força muscular. Para além disso, pretende-se com este trabalho estabelecer, tendo por base a literatura, alguns princípios e regras fundamentais para a prescrição do treino de força em idosos.

Palavras-chave: envelhecimento, treino força, funcionalidade.

\begin{abstract}
Aging and muscle strength - a brief review

Elderly population is growing both in absolute and in percentage of the total population. As the number of elderly people is increasing, recent research is focusing now on how to obtain a better quality of life at older ages. Coping with different daily activities is determined, to a large extent, by muscular strength $(15,52)$. The reduction in muscle strength is a major component of normal aging and is well documented (98). Besides referring the age-related muscular atrophy and weakness, several studies have been performed in order to evaluate the potential influence of physical activity on strength. A number of studies have investigated the effectiveness of physical activity interventions on the maintenance and/or improvement of the muscular strength in elderly people (for refs. see 5). However, these benefits associated with exercise are only of value if physical activity is based on certain rules and principles. Participation in non-systematic and no organized physical activity, especially on strength training, can be dangerous and deleterious for cardiovascular and skeletal muscle systems. In this way, the aim of this paper is to resume the current scientific knowledge concerning muscle aging, its influence on quality of life and the effectiveness of physical activity programs on strength of elderly people. Moreover, this review provides effective guidelines for designing strength fitness prescription programs for older adults.
\end{abstract}

Key Words: aging, strength training, functional ability. 


\section{INTRODUÇÃO}

A população idosa é, actualmente, uma realidade demográfica cada vez mais significativa na população mundial. Por exemplo, em Portugal constata-se que o grupo de idosos, que em 1960 representava 8\% da população, viu esse valor subir para $11.4 \%$ em 1981 e para $14 \%$ em 1991. Em 1997, e de acordo com a estimativa intercensitária da população portuguesa divulgada pelo INE (56) para esse ano, este grupo de pessoas com idade superior a 65 anos passa a corresponder a $15 \%$ da população (103).

Deste modo, não é de estranhar o crescente interesse, particularmente nas últimas décadas, que se tem vindo a observar por parte de investigadores de diferentes ramos do conhecimento pelo bem-estar, saúde e qualidade de vida dos idosos.

O envelhecimento tem sido descrito como um processo, ou conjunto de processos, inerente a todos os seres vivos e que se expressa pela perda da capacidade de adaptação e pela diminuição da funcionalidade (112). O envelhecimento está, assim, associado a inúmeras alterações com repercussões na funcionalidade, mobilidade, autonomia e saúde desta população e, deste modo, na sua qualidade de vida.

Neste sentido, e em termos de saúde pública, interessa sobretudo conhecer as formas de tentar atenuar esta degeneração progressiva. Ao aumento da longevidade deve corresponder a manutenção da qualidade de vida associada à melhor saúde, ao bemestar e à capacidade de realizar autonomamente as tarefas quotidianas (112). Para além dos aspectos directamente relacionados com a saúde, é hoje entendido como tarefa prioritária o desenvolvimento de competências que permitam ao idoso realizar as suas tarefas básicas diárias independentemente do auxílio de terceiros (7). Assim, e dado que a qualidade de vida está intimamente associada a um bom desempenho motor, a prática regular de actividade física torna-se fundamental para este escalão etário. Para manter a qualidade de vida e lidar com as actividades quotidianas, é importante para o idoso permanecer com a melhor aptidão física possível. As actividades diárias, tais como, ir às compras, levantar de uma cadeira, vestir, etc., requerem um nível mínimo de força muscular, coordenação, flexibilidade e equilíbrio $(1,15)$. A capacidade de realizar diferentes actividades diárias, actividades laborais ou recreacionais é determinada, em grande parte, pela capacidade de desenvolver força muscular $(15,52)$. Diferentes autores têm referido que a atrofia e fraqueza musculares associadas ao envelhecimento são aspectos determinantes na morbilidade e mortalidade destes escalões etários mais velhos $(15,21)$. A perda da força e da massa muscular predispõe os idosos a uma limitação funcional, sendo este um factor predisponente para muitos dos processos patológicos associados ao aumento da morbilidade e mortalidade (15).

Ao longo dos anos, um número crescente de estudos tem tentado analisar a potencial influência da actividade física na idade biológica, capacidade funcional e saúde do idoso (para refs. ver 7). Por exemplo, diferentes estudos têm demonstrado que o declínio físico e funcional associado ao envelhecimento pode, mesmo em sujeitos com idade extrema, ser revertido ou, pelo menos, minimizado através do exercício físico $(2,16,18,38)$. Para além disso, sabe-se também que a prática de actividades físicas está associada à redução da incidência de doenças cardiovasculares $(59,69)$, hipertensão (47), diabetes tipo II (3, $22,23,63)$, neoplasia do intestino $(66,121)$, bem como, de estados de ansiedade e depressão (62, 109). Para além disso, a prática regular de actividade física tem sido relacionada com o aumento do conteúdo mineral ósseo e com a redução do risco de fracturas $(61,76,83)$

Neste sentido, o objectivo deste trabalho é rever a literatura sobre o envelhecimento muscular, a sua influência na qualidade de vida e a efectividade dos programas de actividade física nesta capacidade física. Para além disso, dado saber-se que para além dos potenciais benefícios do exercício físico, existem também riscos associados a essa exercitação, uma das preocupações evidentes deste trabalho de revisão foi o de, tendo por base a literatura, fornecer alguns princípios e regras fundamentais para a prescrição do treino de força em idosos.

\section{ENVELHECIMENTO MUSCULAR}

Há mais de 150 anos atrás, Quetelet (93) descreveu originalmente a diminuição da função muscular com o envelhecimento. Desde essa data até à actualidade, vários estudos têm-se ocupado com esta temática, sendo consensual que este decréscimo se torna mais evidente a partir dos 60 anos (30), para além de ser 
mais pronunciado nas mulheres (101).

De acordo com vários autores, a força muscular máxima é alcançada por volta dos 30 anos, mantémse mais ou menos estável até à $5^{\mathrm{a}}$ década, idade a partir da qual inicia o seu declínio. Entre os 50 e os 70 anos existe uma perda de aproximadamente $15 \%$ por década, após o que a redução da força muscular aumenta para $30 \%$ em cada 10 anos (Fig. 1; 70, 98).

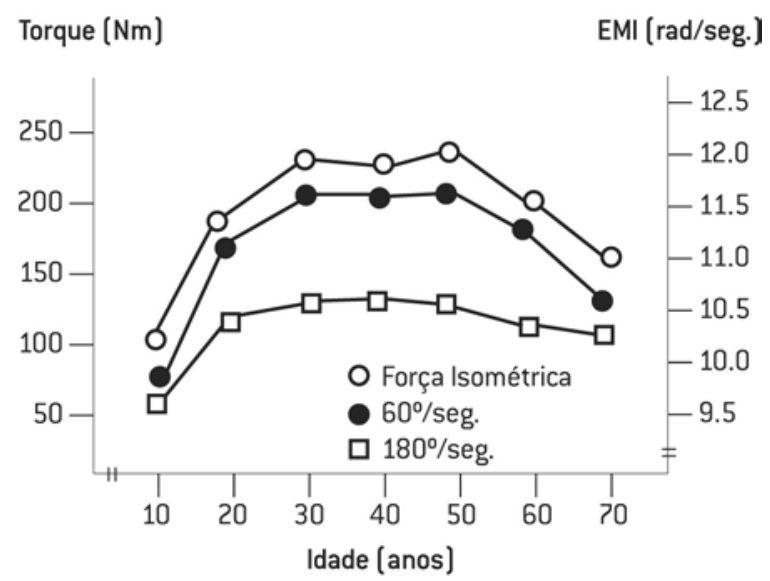

Figura 1 - Força máxima isométrica (FMI, Nm) e velocidade máxima de extensão do joelho [EMJ, rad/seg] em sujeitos do sexo masculino de diferentes grupos etários (adaptado de 66).

A diminuição da força é não apenas específica de cada indivíduo, mas também de cada grupo muscular e ainda do tipo de contracção $(52,112)$. Por exemplo, diferentes estudos mostram que a diminuição da força dos membros inferiores com a idade é mais acentuada do que a observada nos membros superiores (46, 52, 57, 65, 75).

Actualmente está bem descrito na literatura que, quer a massa, quer a força muscular diminuem com a idade $(52,70,75,98)$. Neste sentido, para além da osteoporose e das suas consequências, a sarcopenia que ocorre com o envelhecimento é também um factor importante na saúde do sistema muscular esquelético.

A diminuição da força é atribuída maioritariamente à perda de massa muscular, seja pela atrofia, seja pela redução do número de fibras musculares $(41,73)$. Para além da literatura descrever a atrofia muscular induzida pela idade em diferentes grupos musculares (para refs. ver 88), alguns dados referem ainda um aumento de tecido não contráctil com influência directa no declínio da força observado com o envelhecimento $(72,84)$.

A atrofia das fibras observada no músculo envelhecido inicia-se por volta dos 25 anos com uma diminuição progressiva da área em cerca de $10 \%$ até perto dos 50 anos. Após esta idade, ocorre uma atrofia mais pronunciada, de tal modo que aos 80 anos o idoso sofre uma perda de cerca de $50 \%$ na área de secção transversal do músculo $(73,90)$. Esta atrofia é preferencialmente observada nas fibras tipo II, existindo uma redução média de cerca de $26 \%$ entre os 20 e os 80 anos (73).

No que se refere ao número de fibras musculares, embora exista consenso relativamente à hipoplasia muscular com o envelhecimento, o mesmo não acontece no que respeita ao tipo de fibras que são perdidas.

Para além da atrofia e da hipoplasia, vários trabalhos têm sugerido existir, com o avançar da idade, reduções da capacidade de recrutamento neural, mecanismo que poderá também contribuir de forma significativa para as alterações funcionais observadas nos idosos $(50,119)$. Por exemplo, existem evidências directas e indirectas de alterações quantitativas e qualitativas das UM com a idade (para refs. ver 102). Embora não exista consenso na literatura, vários autores têm descrito alterações com a idade nas propriedades contrácteis (tempo para alcançar pico máximo - TPM, semi-tempo de relaxamento $-1 / 2$ TR, velocidade máxima de encurtamento - Vmax, torque máximo) de diferentes grupos $(116,117)$ Assim, torna-se evidente que o declínio da força com a idade é multifactorial, não podendo ser explicado exclusivamente pela perda da massa muscular (50). Para além dos mecanismos atrás referenciados, outra das possibilidades implicada neste processo é o facto dos idosos terem uma reduzida capacidade de activar completamente os seus grupos musculares (122). Este declínio quantitativo e qualitativo na funcionalidade e estrutura do sistema muscular tem implicações significativas na capacidade funcional do idoso (15). Vários estudos têm demonstrado uma correlação positiva da força muscular, particularmente a força dos extensores do joelho, com a velocidade de marcha $(11,38,67)$, com a subida de degraus (11), com a capacidade de se levantar de uma cadeira (55) 
e com a capacidade de realizar diferentes actividades do dia a dia $(11,55)$.

No estudo de Avlund et al. (11), os idosos (idade média de 75 anos) que apresentavam reduzidos níveis de força nos músculos extensores do joelho, apresentaram também uma maior fatigabilidade, bem como uma maior necessidade de ajuda na realização de diferentes actividades diárias.

Para além deste facto, a literatura sugere que os baixos índices de força estão relacionados com a maior susceptibilidade de ocorrência de quedas e consequentes fracturas, facilitadas pela desmineralização óssea comum neste escalão etário $(1,21,31)$.

Embora ainda não tenha sido estabelecida uma relação de causa-efeito entre a força muscular e a incidência de quedas, diferentes estudos suportam esta hipótese $(74,97)$. Comparativamente ao grupo controlo da mesma idade, os idosos com história de quedas frequentes apresentaram valores significativamente mais baixos na força e potência muscular dos quatro principais grupos musculares implicados no equilíbrio, i.e., flexores e extensores do joelho, extensores e flexores do pé (95).

As quedas são hoje consideradas um problema de saúde pública, pois para além dos custos sociais que lhes estão inerentes, implicam, na sua maioria, o recurso à situação de acamamento e, deste modo, a uma aceleração da senescência do idoso (29).

Vários trabalhos mostram que 40 a $60 \%$ dos indivíduos acima dos 65 anos já experimentaram pelo menos uma queda, sendo esta mais frequente nos utentes de lares e nas mulheres $(107,108)$. Apesar de se estimar que apenas $10 \%$ das quedas resultam em fractura óssea grave, aproximadamente $20 \%$ das mulheres que sofrem uma fractura da anca, não sobrevivem para além do primeiro ano e outras $20 \%$ ficam com uma limitada mobilidade e dependentes de outros (105).

O equilíbrio é outra capacidade determinante para a funcionalidade e saúde dos idosos que, para além de outros aspectos, também depende em grande escala da força dos membros inferiores (21).

A manutenção do equilíbrio, quer estático, quer dinâmico relaciona-se com diferentes factores. A deterioração da visão, do sistema vestibular e somatosensorial que decorrem do próprio processo de envelhecimento, constituem-se como importantes causas para a afectação do equilíbrio (112).

O equilíbrio diminui com o envelhecimento, verificando-se um declínio mais acentuado a partir da $6^{a}$ década. Não apenas a frequência e a amplitude da oscilação corporal é maior nos idosos, comparativamente aos jovens, como também a correcção da estabilidade corporal é mais lenta nos escalôes etários mais velhos (para refs. ver 29).

Por outro lado, as alterações degenerativas da coluna, conjuntamente com a diminuição heterogénea da força e/ou com diminuição da flexibilidade a este nível, resultam numa maior curvatura cifótica, o que também desfavorece o equilíbrio. Com o envelhecimento, os discos intervertebrais tornam-se progressivamente mais achatados e menos elásticos e as vértebras adquirem gradualmente, por processos osteoporóticos, a forma de cunha originando o desalinhamento compensatório das vértebras dorsais e cervicais (112). Actividades como caminhar, subir degraus, levantar-se de uma cadeira, podem induzir um "stress" mecânico evidente sobre estas vértebras mal posicionadas, resultando na exacerbação da dor. Por seu lado, longos períodos de inactividade, particularmente na posição de sentado, aumentam a curvatura da zona cervical, ombros e zona lombar com aumento da degeneração da coluna vertebral, aumento da dor e diminuição da mobilidade (112). Por outro lado, as diferentes patologias cardiovasculares e alterações neuromusculares, bem como a acção de fármacos, particularmente os que se referem à acção do sistema nervoso central, podem também contribuir para aumentar a instabilidade corporal (21). A diminuição da força muscular, particularmente dos membros inferiores, relaciona-se não apenas com o declínio do equilíbrio mas igualmente com a qualidade da marcha $(67,96)$.

Assim, e dado que a locomoção é basicamente um processo de transferência do centro de gravidade de um pé para outro, numa série de sucessivas perdas de equilíbrio, é natural que seja influenciada pelo envelhecimento.

A característica mais evidente da marcha do idoso é a sua maior lentidão, ou seja, com a idade a marcha torna-se progressivamente mais lenta, sendo este aspecto mais pronunciado entre os 65 e os 85 anos e mais evidente nas mulheres $(29,58)$. Tanto os jovens como os idosos alteram o comprimento e a 
frequência de passada, de forma a modificar a velocidade da mesma. Contudo, enquanto os idosos tendem a favorecer o aumento da cadência, os jovens preferem aumentar o comprimento da passada (36). Provavelmente, os idosos não optam por aumentar o comprimento da passada, já que, para além de menos flexíveis, o seu equilíbrio está comprometido e, como tal, eles privilegiam uma maior permanência dos dois pés em contacto com o solo. Aumentar o comprimento da passada implica uma diminuição do tempo em que ambos os pés se encontram apoiados no solo, acto que requer mais força e maior equilíbrio (112). Outro factor importante para as alterações observadas na passada é a economia de movimentos.

Possivelmente, os idosos adoptam a sua velocidade de marcha de forma a serem mais económicos, tendo por base a sua estrutura corporal, peso, força e resistência (58). Assim, o padrão de passada do idoso é mais lento e a sua passada é mais curta existindo uma menor relação entre o tempo de balanço e o tempo de apoio. Ou seja, existe um aumento da fase de duplo apoio e uma consequente diminuição da fase de balanço, com diminuição do comprimento do passo. Outra característica da marcha do idoso é a menor elevação do calcanhar relativamente ao solo $(58,120)$.

\section{TREINO DE FORÇA E ENVELHECIMENTO}

Dado que, tal como referido anteriormente, a fraqueza muscular contribui para alterações na mobilidade, autonomia, bem como, para o maior risco de quedas e fracturas nos idosos, um adequado programa de treino de força pode constituir-se como um meio importante para a vida diária do idoso.

Níveis moderados de força são necessários para a realização de inúmeras tarefas diárias, tais como, carregar pesos, subir escadas, levantar-se de cadeiras, etc. Neste sentido, a força adquire uma importância cada vez mais acentuada com o avançar da idade (15).

Fiatarone et al. (37) demonstraram existir uma relação inversa entre a força dos músculos extensores do joelho e o tempo de marcha em 6 metros em idosos (86 e 96 anos) de ambos os sexos. De igual modo, Sipila et al. (110) encontraram uma velocidade máxima da marcha significativamente mais elevada, quer após 18 semanas de treino de força, quer após 18 semanas de treino de resistência em mulheres idosas entre os 76 e os 78 anos.
Mesmo em sujeitos mais debilitados, têm sido encontrados aumentos de força e da área muscular com consequente melhoria funcional. Fiatarone et al. (38) observaram após treino de força de elevada intensidade, alterações positivas na mobilidade (velocidade de marcha e velocidade de subir/descer degraus) e na actividade física espontânea, em idosos residentes de lares entre os 72 e 98 anos.

Todavia, decorridas as 10 semanas de treino, os aumentos em termos percentuais das alterações funcionais ( 8 a $51 \%$ ) foram bastante menores do que os ganhos de força (26 a 215\%). Ou seja, embora os ganhos de força sejam estatisticamente significativos, quando analisados sob o ponto de vista clínico a sua importância não se torna tão evidente.

No entanto, mais recentemente, Chandler et al. (24) encontraram pequenos mas significativos aumentos na força muscular $(\sim 11 \%)$ associados a melhorias na funcionalidade e mobilidade, sugerindo que para aumentar a capacidade funcional diária não são necessários aumentos substanciais da força. Uma pequena activação muscular é provavelmente suficiente para reduzir a fragilidade muscular típica do idoso.

Para além dos ganhos de força, os programas de treino desta capacidade física aumentam a coordenação neuromuscular e a potência (1). A preservação da coordenação e da potência muscular em idades avançadas pode diminuir significativamente o risco de queda e aumentar a independência funcional (33). Campbell et al. (20) encontraram, após a aplicação de um programa de actividade física englobando, fundamentalmente, exercícios de força para os membros inferiores e exercícios de equilíbrio e marcha (30 min/dia, 3 x sem), uma redução significativa no número de quedas em sujeitos com média de idade de 80 anos comparativamente ao grupo controlo de idade semelhante. De igual modo, Buchner et al. (18) descreveram um menor número de quedas em idosos submetidos a treino combinado de força, resistência e flexibilidade.

Numerosos estudos têm demonstrado que estímulos adequados de treino de força em homens e mulheres idosas, promovem ganhos da força similares ou até superiores aos encontrados em jovens $(26,37,42$, 92). Frontera et al. (42) observaram um aumento de cerca de $100 \%$ da repetição máxima (1RM) na força dos extensores do joelho e acima dos $200 \%$ nos fle- 
xores, após 12 semanas de trabalho intenso de força dinâmica ( $80 \%$ de 1RM; 3 séries x 8 reps.; 3 x/sem) em idosos homens entre os 60 e 72 anos. De igual modo, estudos mais recentes observaram aumentos de $100 \%$ (1RM) após 2-3 meses de treino, quer em homens, quer em mulheres idosas $(26,38)$. São vários os mecanismos que podem explicar os ganhos da força com treino intenso, incluindo alterações bioquímicas e morfológicas musculares, alterações na biomecânica do tecido muscular e conjuntivo, activação do sistema nervoso central, coordenação motora e aspectos psicológicos (87).

Do ponto de vista morfológico, o músculo envelhecido apresenta uma plasticidade semelhante à encontrada no jovem (98). Frontera et al. (42) para além dos ganhos de força muscular, quer nos extensores (107\%), quer nos flexores (227\%) do joelho, encontraram, após 12 semanas de treino de força em sujeitos com idade média de 66 anos, um aumento significativo de cerca de $11 \%$ na área total muscular (avaliada por tomografia axial computadorizada - TAC) com aumento do "turnover" proteico (avaliado pela excreção urinária de 3-metil-histidina). Ainda neste estudo, biópsias do m. vastus lateralis revelaram aumentos da área das fibras I e II de, respectivamente, $34 \%$ e $28 \%$.

Outros estudos utilizando TAC têm demonstrado que o treino de força intenso resulta numa significativa hipertrofia muscular mesmo em sujeitos mais velhos com idades compreendidas entre os 86 e os 98 anos $(16,37)$. Mais recentemente, a ressonância magnética nuclear (RMN) tem também confirmado estes resultados (100).

Assim, numerosos estudos confirmam a elevada plasticidade muscular no idoso em resposta ao treino da força. Por exemplo, Pyka et al. (92) estudaram o efeito do treino da força em 8 homens e 17 mulheres com uma média de idade de 68 anos. Um aumento significativo na área de secção transversal das fibras musculares tipo I foi observado após um ano de treino de força ( $75 \%$ de 1RM; 3 séries; 8 reps.; $3 \mathrm{x} \mathrm{sem}$ ). No final do programa, a força aumentou cerca de $30 \%$ nos extensores e $97 \%$ nos flexores da anca. Este aumento da força foi mais evidente nos primeiros três meses, após o qual se manteve até ao final do programa. A área de secção transversal das fibras I aumentou em média $25 \%$ e
$48 \%$ decorridas 15 e 30 semanas, respectivamente. A área das fibras II não se alterou significativamente após 15 semanas tendo, no entanto, aumentado cerca de $62 \%$ no final das 30 semanas. Os autores concluíram que o treino prolongado de força de moderada a elevada intensidade pode ser efectuado, com elevada tolerância, por sujeitos idosos com consequente adaptação funcional e morfológica.

Por outro lado, paralelamente às adaptações neurais e morfológicas musculares, as características intrínsecas musculares, tais como processos de excitação/contracção, densidade de agrupamento de fibras musculares e a composição das fibras, podem alterar a produção de força (14).

Mesmo em sujeitos mais idosos têm sido encontrados aumentos da força acompanhados por alterações electromiográficas importantes. Assim, Grimby et al. (46) submeteram 9 idosos do sexo masculino (78-84 anos) a 25 sessões de treino de força (2-3 x semana) com diferentes tipos de contracção muscular (concêntrica, excêntrica e isométrica). No final do programa foi observado um significativo aumento da força muscular e elevações da actividade electromiográfica, permitindo inferir da elevada treinabilidade do músculo envelhecido, tanto em termos funcionais, como electrofisiológicos.

De igual modo, Rice et al. (94) estudaram o efeito de 24 semanas de treino ( $80 \%$ de 1 RM, 4 séries de $6-8$ reps.) no $m$. triceps brachii em sujeitos com idades compreendidas entre os 65 e os 78 anos. No final do programa, a força máxima aumentou $20 \%$ e o tempo para alcançar pico máximo (TPM) foi elevado em $11 \%$ sem, no entanto, se terem observado alterações nas restantes variáveis contrácteis (p.ex: semi-tempo de relaxamento $-1 / 2 \mathrm{TR}$ ). Estes resultados demonstram, tal como no trabalho de Grimby et al. (46), que para além do aumento da capacidade funcional, o músculo envelhecido manifesta também profundas adaptações nas suas características eléctricas e contrácteis.

Klitgaard et al. (64) descreveram adaptações significativas na função, morfologia e na composição das cadeias leves e pesadas de miosina, bem como, na relação $\beta / \alpha$ da tropomiosina em idosos praticantes de halterofilia. Também, Trappe et al. (118) ao estudarem o efeito de 12 semanas de treino de força na função contráctil de fibras musculares isoladas do $m$. vastus lateralis, verificaram que, quer as cadeias pesa- 
das de miosina tipo I, quer as tipo IIa se tornaram mais potentes, produzindo maiores picos de tensão e contraindo-se mais rapidamente. Assim, o treino da força regular parece não apenas estimular a força e a hipertrofia muscular em idosos, como também induzir alterações nas suas propriedades contrácteis. Embora os mecanismos responsáveis pelo aumento dos níveis de força com o treino não estejam completamente esclarecidos, parece que o aumento da frequência de disparo das unidades motoras (UM) e do seu recrutamento máximo, são também possíveis factores influenciadores do aumento da força após curtos períodos de treino (para refs. ver 54).

Paralelamente, o aumento da eficácia das UM parece ser um possível mecanismo para explicar o aumento da capacidade dos idosos em manterem contracções submáximas (68).

Resumindo, diferentes estudos demonstram que as pessoas idosas são capazes de melhorar a sua capacidade de desenvolver força. Estes aumentos da força muscular, parecem, por seu lado, estar associados a melhorais funcionais, aspecto determinante para a manutenção da autonomia diária do idoso e consequentemente para a sua melhor qualidade de vida. Para além dos factores mais relacionados com a funcionalidade, o treino de força parece ajudar a manter ou até melhorar a densidade mineral óssea (61, 76, 83), a taxa metabólica basal (19), a sensibilidade da insulina $(32,82)$, o tempo de trânsito intestinal e a diminuir a dor e a incapacidade induzidas pela degeneração articular (para refs. ver 60).

Para além destes efeitos, apesar de controversos, parece que o treino da força, ao aumentar a massa magra e a actividade do sistema nervoso simpático (SNS), aumenta também a taxa de metabolismo basal, podendo contribuir para a redução da massa gorda (89), diminuindo assim, os factores de risco de acidente cardiovascular (para refs. ver 79).

Relativamente aos efeitos do treino de força sobre as adaptações cardiovasculares, a literatura, contrariamente aos efeitos do treino de resistência aeróbia, é relativamente escassa.

Por outro lado, mesmo os estudos que encontram aumentos significativos no consumo máximo de oxigénio $\left(\mathrm{VO}_{2} \mathrm{max}\right)$ após treino de força $(43,48)$, referem que a magnitude destes ganhos $(-5 \%)$ não é apreciável quando comparada com os efeitos do trei- no de resistência aeróbia. Assim, a literatura indica que o treino da força não aumenta significativamente o $\mathrm{VO}_{2}$ max para além da normal variação biológica e metodológica (54).

No entanto, apesar de não serem observadas melhorias substanciais na capacidade máxima aeróbia, o treino de força poderá induzir adaptações que beneficiarão o sistema cardiovascular dos idosos. Por exemplo, Parker et al. (85) descreveram após 16 semanas de treino de força, diminuições da frequência cardíaca e da pressão arterial em teste submáximo de marcha em tapete em sujeitos entre os 60 e os 77 anos. Assim, apesar das pequenas alterações no $\mathrm{VO}_{2} \mathrm{max}$, o treino de força pode aumentar a aptidão cardiovascular submáxima. Os possíveis mecanismos para estas adaptações na performance submáxima cardiovascular serão as alterações no recrutamento do tipo de fibras (i.e., maior percentagem de fibras tipo I recrutadas), a melhor redistribuição do fluxo sanguíneo e o aumento do limiar anaeróbio (77).

Assim, apesar de ligeiras, as adaptações na aptidão cardiovascular submáxima induzidas pelo treino de força são importantes na medida em que diminuem o risco de acidente cardiovascular (113). De acordo com Stone et al. (113), ao aumentar a força máxima com o treino, o idoso vai, perante uma dada tarefa submáxima, requerer um menor esforço e, assim, induzir um menor "stress" cardiovascular.

Apesar dos dados da literatura não serem conclusivos quanto ao efeito do treino de força na redução dos valores da pressão arterial em idosos hipertensos, este facto é evidente em sujeitos normotensos (85). Por fim, embora os efeitos do treino de força na alteração da tolerância à glicose não sejam unânimes, a maioria dos estudos demonstram, mediante a redução das respostas insulínicas dos testes orais de tolerância à glicose, que um programa de treino de força adequado pode melhorar a acção da insulina e, assim, favorecer os sujeitos portadores de diabetes tipo II (para refs. ver 54).

Apesar do exercício aeróbio ser aquele que, tradicionalmente, é o mais recomendado para aumentar a aptidão física, o treino de força é também, hoje, considerado um componente fundamental do programa geral de actividade física. O ACSM (5) refere-se à inclusão do treino de força como parte integrante do programa de actividade física do idoso. 
A importância do treino da força como parte integrante de um programa de actividade física para idosos foi demonstrada por Klitgaard et al. (64). Estes autores, ao estudarem a função, morfologia e expressão das cadeias de miosina em jovens e idosos com diferentes regimes de treino (sedentários, halterofilistas, fundistas e nadadores), verificaram que, quer os nadadores, quer os fundistas apresentavam valores no momento máximo isométrico, área de secção transversal e composição das isoformas das cadeias leves e pesadas de miosina do $\mathrm{m}$. vastus lateralis semelhantes ao grupo controlo com a mesma idade. Apenas os idosos halterofilistas apresentaram adaptações significativas relativamente aos sedentários, sendo os seus valores aproximados do grupo controlo jovem. De igual modo, somente os idosos halterofilistas apresentaram um perfil da tropomiosina (relação $\beta / \alpha$ ) idêntico aos jovens sedentários. Estes resultados apontam para uma especificidade dos efeitos do treino de força na função muscular, sugerindo que o treino de resistência cardiovascular, por natação ou corrida, não atenua os declínios funcionais, morfológicos e contrácteis associados ao envelhecimento.

Assim, tendo por base a configuração de todos os argumentos anteriormente referidos, o desenvolvimento de estratégias de preservação e/ou aumento da massa e força musculares de idosos sedentários, constitui-se como um meio importante de aumentar a independência funcional e diminuir a prevalência de algumas doenças crónicas comuns neste escalão etário. Diferentes estudos têm demonstrado que, independentemente da idade e do sexo, com estímulos adequados de treino é possível aumentar a força muscular dos músculos exercitados $(49,71)$.

A intensidade do treino parece ser um aspecto determinante, correspondendo as mais elevadas intensidades de treino a maiores adaptações. De um modo geral, a literatura considera intensidades elevadas as realizadas acima de $80 \%$ de $1 \mathrm{RM}$. As realizadas a 50\%-60\% de 1RM são classificadas como sendo de moderada intensidade e, por fim, as inferiores ou iguais a $40 \%$ de 1 RM são consideradas de baixa intensidade (115).

Estudos recentes recomendam uma intensidade de $80 \%$ de 1RM para maximizar a força e os ganhos funcionais após treino de força $(33,37,46,83,115)$.
Todavia, por outro lado, existem também trabalhos que descrevem que baixas intensidades e mesmo baixas frequências de treino de força induzem, não apenas melhorias na força $(2,68,91,115)$, como também podem aumentar a funcionalidade dos idosos (24). Por exemplo, Pruitt et al. (91) encontraram aumentos de, respectivamente, 42 e $48 \%$ nos níveis de força nos membros inferiores ("leg press") após 12 meses de treino de força, tanto com baixas (40\% de $1 \mathrm{RM})$, como com elevadas intensidades $(80 \%$ de 1RM). Assim, parece que, não apenas a intensidade da contracção per se (80), mas também a carga total ou a prática de determinado movimento (68) podem aumentar a força máxima. Estudos utilizando estas baixas a moderadas intensidades têm descrito aumentos na ordem dos 10 a $30 \%$ da força em idosos saudáveis e independentes na sua vida diária $(17,24)$. Connelly e Vandervoort $(27)$, ao submeterem 10 mulheres idosas $(81.6 \pm 8.4$ anos $)$ a 8 semanas de treino de força com intensidades entre os 30 e os $50 \%$ de $1 \mathrm{RM}$ ( 3 x sem), mostraram que não são necessárias elevadas intensidades de treino de força para induzir aumentos nesta capacidade.

Recentemente, Bemben et al. (12) mostraram que um programa de baixa intensidade com volume de treino suficiente, pode produzir ganhos de força relativos semelhantes ao treino de elevada intensidade em mulheres pósmenopausa sedentárias.

Neste sentido, e de acordo com Fiatarone et al. (38), é clinicamente importante perceber que, se um idoso não tolera elevadas intensidades como consequência da dor articular ou de qualquer outra enfermidade, um programa modificado de exercício constitui-se como uma alternativa importante para a melhoria da saúde e da aptidão física. O menor desconforto e a menor probabilidade de lesão, tornam o exercício de baixa a moderada intensidade uma alternativa viável para aumentar a força muscular. Estudos com intervenções comportamentais no sentido de aumentar a actividade física de idosos sedentários, têm referido uma maior aderência dos sujeitos aos programas de exercício físico de mais baixa intensidade. Assim, numa fase inicial, o começar com intensidades mais baixas pode ser um requisito importante para posteriormente ser possível aumentar as cargas de treino. $\mathrm{O}$ treino de força deverá ser progressivo, individualizado, induzindo estímulos para os principais grupos 
musculares envolvidos nas actividades do dia a dia (5). A inclusão de um período de familiarização é fundamental, particularmente nestes escalões etários mais velhos já que a introdução de novas habilidades implica uma aprendizagem que, geralmente, é mais lenta neste escalões etários mais velhos. De um modo geral, os idosos necessitam de um período de tempo mais alargado para se adaptarem aos regimes rigorosos do treino e, como tal, para optimizar os benefícios do treino é importante considerar a existência de um maior período de adaptação.

Para além disso, esse período inicial de familiarização permite dispor de um período de tempo para efectuar correcções posturais e de execução enquanto o estímulo é menos intenso. A maioria das lesões registadas nos estudos com este tipo de trabalho ocorrem nas primeiras duas semanas por falta de técnica de treino (106). Para além disso, muitos dos aumentos iniciais na força que ocorrem com treino são devidos a alterações neuromusculares que resultam numa melhoria no recrutamento das fibras musculares (72).

O volume de treino é igualmente um aspecto importante para optimizar a resposta de adaptação ao treino de força; se a intensidade do treino for baixa, o número de repetições e o volume total de treino devem ser médios a grandes, de forma a maximizar a resposta muscular ao treino de força.

Vários autores recomendam uma frequência de 2 a 3 dias por semana, 8 a 10 exercícios, 2 a 3 séries de 8 a 12 repetições cada (para refs. ver 79). Estas recomendações são baseadas em 3 factores principais: 1) o tempo que demora a completar um programa abrangente de exercícios de força. Programas acima dos 60 minutos por sessão parecem estar associados a elevadas taxas de abandono (35). Merssier e Dill (81) referem que o tempo médio requerido para completar três séries ( 8 a 10 reps.) é de cerca de 50 minutos comparativamente a apenas 20 minutos para uma série; 2) apesar das maiores frequências e do maior número de séries ou combinação de séries e repetições poderem induzir maiores ganhos de força (40), as diferenças nas melhorias na aptidão geral do idoso são, geralmente, reduzidas; 3) apesar de com cargas elevadas (1-6RM), poucas repetições e múltiplas séries se obterem maiores ganhos na força e na massa muscular, esta abordagem pode não ser a melhor para os sujeitos com objectivos diferentes dos dos atletas. Para além disso, do ponto de vista da segurança, este tipo de programas pode aumentar o risco de lesão ortopédica e precipitar algum problema cardíaco nos sujeitos idosos (86). Para induzir melhorias na força e resistência muscular, a maioria dos autores sugere 8 a 12 repetições por série (5). No entanto, e porque a lesão no aparelho muscular esquelético pode ocorrer nos mais velhos e mais debilitados, o ideal é utilizar cargas baixas a moderadas com 10 a 15 repetições $(5,33,79)$. Por outro lado, os exercícios de força devem ser realizados na sua amplitude máxima, de forma lenta e controlada, e acompanhada por uma respiração ritmada, evitando sempre o bloqueio respiratório (manobra de Valsalva) dada a sua influência na elevação dos valores da pressão arterial (33). Fleck et al. (39), mostraram existir uma relação directa entre a pressão intratorácica provocada pela manobra de Valsalva e os valores da pressão arterial sistólica (PAS), diastólica (PAD), débito cardíaco e volume sistólico durante a realização de exercícios de força. Mais recentemente, Bermon et al. (13), ao avaliarem a tolerância de idosos saudáveis ao treino de força, verificaram que, mesmo quando os valores mais elevados da frequência cardíaca (FC), PAS e PAD foram alcançados durante a realização dos exercícios bilaterais máximos, em nenhum caso, o treino de força aumentou significativamente os valores da troponina cardíaca sérica (cTnI). Como vem sendo descrito (13), esta proteína tem sido relacionada com lesão cardíaca e, deste modo, como um indicador indirecto de sobrecarga miocárdica. Assim, de acordo com estes autores a tolerância cardiovascular ao treino de força é boa, desde que sejam cumpridos os critérios de selecção e forem realizadas as técnicas correctas de respiração, evitando sempre a manobra de Valsalva.

Tal como referido anteriormente, para obter o máximo benefício, os exercícios de força devem ser realizados na amplitude máxima individual. A especificidade do treino quanto à área corporal e amplitude de movimento envolvida está bem descrita na literatura $(9,40,45)$. Por exemplo, Graves et al. (45) no sentido de estudarem o efeito da amplitude do movimento sobre a força dos músculos extensores do joelho, dividiram a sua amostra em 4 grupos distintos: o grupo 1 que realizou apenas a primeira metade da 
amplitude do movimento; o grupo 2 que efectuou apenas a segunda metade da amplitude do movimento; o grupo 3 que trabalhou o movimento na sua amplitude total e finalmente, o grupo controlo que se absteve de treinar. De acordo com os resultados deste estudo, o treino induz melhorias significativas sobre a força dos músculos dos membros inferiores, sendo, no entanto, este aumento específico do ângulo articular treinado, com o grupo 3 a obter o melhor resultado na amplitude total do movimento. Estes resultados sugerem que o treino de força deve ser realizado na amplitude máxima individual para obter os máximos benefícios (45). Por outro lado, embora controverso, é geralmente assumido que um treino de força apropriado com realização da amplitude total de movimento e exercitando, quer os músculos agonistas, quer os antagonistas melhora a flexibilidade $(1,2,113)$. A importância da flexibilidade do idoso é inegável, não apenas em termos funcionais, como também, está relacionada com parâmetros da saúde. Os baixos valores de flexibilidade têm sido associados à maior prevalência de lesões, particularmente da coluna vertebral $(6,113)$, bem como, à maior dificuldade em caminhar e em realizar autonomamente as tarefas diárias $(1,6,113)$.

No entanto, dada a importância da flexibilidade no dia a dia do idoso e dado que esta opinião não é partilhada por outros investigadores - que ou não encontraram diferenças nos valores da flexibilidade entre o grupo treinado com força e o grupo controlo (45) ou descrevem a sua diminuição após treino de força (78) - devem ser incluídos no programa de actividade física exercícios específicos de flexibilidade (44).

A magnitude da resposta ao treino de força depende também, em grande parte, do tipo de treino: pesos livres ou máquinas de resistência variável. A maioria dos estudos da literatura considera o trabalho em máquinas de resistência variável como sendo o ideal, uma vez que permite, não apenas a realização controlada do movimento mantendo uma correcta postura, como também, permite ajustar a carga mais apropriada ao grupo muscular e indivíduo em causa, obedecendo, assim, ao princípio da sobrecarga (79). Por outro lado, e de acordo com Hill e Piper (51), o uso de treino em circuito, não apenas permite uma melhor organização da sessão, como também facilita o entendimento dos idosos quanto à execução dos diferentes exercícios, para além de facilitar o controlo dos tempos de recuperação, aspecto determinante neste escalão etário. A recuperação neste grupo de sujeitos é de extrema importância, pois para além da influência das frequentes patologias cardiovasculares, o sistema cardiovascular é menos eficaz. Neste sentido, para além das patologias comuns neste escalão etário, a idade só por si é um factor modificador importante que devemos considerar na prescrição dos exercícios e nos tempos de recuperação. De facto, as respostas mais exuberantes, quer da FC, quer da PA ao exercício de força, podem, juntamente com as patologias cardiovasculares características deste escalão etário, aumentar significativamente o "stress" cardiovascular e, consequentemente, aumentar o factor de risco associado à exercitação $(84,111)$. É importante salientar que nos países industrializados cerca de $50 \%$ dos sujeitos entre os 60 e os 70 anos sofrem de hipertensão (53), e que o exercício, particularmente o de força, induz, por si só, aumentos da pressão arterial $(8,60)$.

Neste sentido, durante estas sessões de trabalho de força, e com o propósito de minimizar a fadiga sem sobrecarregar o sistema muscular e cardiovascular, não apenas se deve trabalhar de forma alternada a parte inferior e superior do corpo, como também, os intervalos entre as séries devem permitir a recuperação completa.

Muito da "não-recomendação" tradicional do treino de força em idosos baseou-se no facto deste tipo de exercícios poder aumentar exageradamente os valores da pressão arterial. Ou seja, apesar do treino de força ser recomendado para este escalão etário, dadas as suas repercussões na funcionalidade, saúde e qualidade de vida do idoso $(23,33,90)$, existe alguma preocupação quanto à segurança deste tipo de exercícios uma vez que a sua componente isométrica pode potenciar arritmias (10) e elevar os valores da pressão arterial (104). Para além disso, muitos dos idosos que se iniciam neste tipo de treino são sedentários e, eventualmente, poderão sofrer de conhecidas ou imperceptíveis patologias cardiovasculares (34).

Actualmente, a literatura defende, no entanto, que quando efectuada com técnicas apropriadas, a elevação dos valores da pressão arterial durante os exercícios de força é apenas ligeira (33). Por exemplo, 
Overend et al. (84) avaliaram o comportamento da FC, PAS e PAD após exercícios isocinéticos de força dos membros inferiores entre jovens (23.4 \pm 1.7 anos) e idosos (75.2 \pm 4.6 anos), não tendo encontrado diferenças com significado estatístico em nenhuma das variáveis. Assim, de acordo com os autores, do ponto de vista cardiovascular, os exercícios de força são bem tolerados pelos idosos, podendo ser utilizados para a sua avaliação, treino e reabilitação.

O tipo de exercício, a intensidade e a duração e, particularmente, a quantidade de massa muscular activa são factores habitualmente relacionados com a magnitude do aumento da FC e da PA $(60,84)$.

Hoje em dia, dado considerar-se uma capacidade determinante para a realização de inúmeras actividades diárias (33), o treino de força, paralelamente às actividades aeróbias, faz cada vez mais parte integrante dos programas de reabilitação cardíaca. Ao contrário do que tradicionalmente era descrito, estudos recentes $(33,99)$ referem que a elevação dos valores da pressão arterial, particularmente os da PAS, durante esforço aeróbio é geralmente maior, comparativamente ao treino de força.

Outra recomendação importante, que deve ser considerada no trabalho de força, refere-se ao equilíbrio entre os músculos flexores e os extensores. De facto, a maioria das actividades exige uma maior solicitação dos músculos extensores em detrimento dos flexores, resultando daí desequilíbrios marcados que se traduzem por fadiga generalizada, sensação de astenia e maior probabilidade de lesão (11). Assim, o trabalho de força deve ser orientado no sentido de existir um equilíbrio entre os músculos flexores e extensores.

Por fim, os benefícios do treino sobre o sistema muscular esquelético são dependentes do carácter contínuo e regular do exercício (4). Por exemplo, Connelly e Vandervoort (28) observaram, após 1 ano de cessação de actividade num grupo de idosas com média de idade de 83 anos submetidas a treino de força durante 8 semanas, uma diminuição da força dos músculos extensores do joelho de cerca de $25 \%$ comparativamente aos valores de pós-treino e de $10 \%$ em relação aos valores de pré-treino.

Paralelamente às alterações na força muscular foram igualmente observadas, no estudo destes autores, alterações na mobilidade funcional após destreino.
Vários estudos têm descrito que as adaptações, quer morfológicas, quer funcionais podem desaparecer mesmo após curtos períodos de destreino. Por exemplo, Taaffe e Marcus (114) descreveram uma perda de $30 \%$ dos ganhos iniciais da força muscular após 12 semanas de destreino na sequência de 24 semanas de treino de força. Para além da função, também a área das fibras tipo I e II foi revertida com o destreino aos valores de pré-treino.

Resumindo, o treino progressivo de força com intensidade moderada, realizado com técnicas apropriadas, pode ser efectuado com elevada tolerância por idosos saudáveis, desempenhando um papel importante enquanto estratégia para a manutenção e/ou aumento da força muscular.

\section{CORRESPONDÊNCIA Joana Carvalho}

Faculdade de Ciências do Desporto

e de Educação Física

Universidade do Porto

Rua Dr. Plácido Costa, 91

4200-450 Porto, Portugal

jcarvalho@fcdef.up.pt 


\section{REFERÊNCIAS BIBLIOGRÁFICAS}

1. Adams K, O'Shea P, O'Shea KL (1999). Aging: its effects on strength, power, flexibility, and bone density. Natl Strength Cond Assoc J 21: 65-77.

2. Adams K, Swank AM, Berning JM, Sevene-Adams PG, Barnard KL, Shimp-Bowerman J (2001). Progressive strength training in sedentary older African American women. Med Sci Sports Exerc 33: 1567-1576.

3. Ades P, Grunvald M (1990). Cardiopulmonary exercise testing before and after conditioning in older cardiac patients. Am Heart J 69: 1442-1446.

4. American College Sports Medicine (1998a). Position stand on exercise and physical activity for older adults. Med Sci Sports Exerc 30: 992-1008.

5. American College Sports Medicine (1998b). Position stand on the recommended quantity and quality of exercise for developing and maintaining cardiorespiratory and muscular fitness, and flexibility in healthy adults. Med Sci Sports Exerc. 30: 975-991.

6. American College Sports Medicine (2001). Resource Manual for Guidelines for Exercise Testing and Prescription $4^{\text {th }}$ Ed. Philadelphia.

7. Andrews GR (2001). Promoting health and function in an ageing population. BMJ. 322: 728-729.

8. Astrand PO (1992). "Why exercise?" Med Sci Sports Exerc 24: 153-162.

9. Astrand PO, Rodahl K (1986). Textbook of work physiology. 3rd Ed. McGraw-Hill Book Co.

10. Atkins JM, Matthews OA, Blomqvist CG, Mullins CB (1976). Incidence of arrythmias induced by isometric and dynamics exercise. Br Heart J 38: 465-471.

11. Avlund K, Schroll M, Davidsen M, Lovborg B, Rantanen T (1994). Maximal isometric muscle strength and functional ability in daily activities among 75 -year-old men and women. Scand J Med Sci Sports. 4: 32-40.

12. Bemben DA, Fetters NL, Bemben G, Nabbavi N, Koh ET (2000). Musculoskeletal responses to high- and low-intensity resistance training in early postmenopausal women. Med Sci Sports Exerc 32: 1949-1957.

13. Bermon S, Rama D, Dolisi C (2000). Cardiovascular tolerance of healthy elderly subjects to weight-lifting exercises. Med Sci Sports Exerc 32: 1845-1848.

14. Blimkie CJR (1992). Resistance training during pre-and early puberty: efficacy, trainability, mechanisms, and persistence. Can J Sport Sci 17: 264-279.

15. Brill PA, Macera CA, Davis DR, Blair SN, Gordon N (2000). Muscular strength and physical function. Med Sci Sports Exerc 32: 412-416.

16. Brown A, McCartney N, Sale D (1990). Positive adaptations to weight-lifting training in the elderly. J Appl Physiol 69: 1725-1733.

17. Brown M, Holloszy JO (1991). Effects of a low intensity exercise program on selected physical performance characteristics of 60 to 71 year olds. Aging Clin Exp Res 3: 129-139.

18. Buchner DM, Cress ME, deLauteur BJ, Esselman PC, Margherita AJ, Price R, Wagner EH (1997). The effect of strength and endurance training on gait, balance, fall risk and health services used in community-living older adults. J Gerontol 52: M218-M224.

19. Campbell AJ, Robertson MC, Gardner MM, Norton RN, Buchner DM (1999). Falls prevention over 2 years: a ran- domized controlled trial in women 80 years and older. Age Aging 28: 513-518.

20. Campbell WW, Crim MC, Dallal GE, Young VR, Evans WJ (1994). Increased protein requirements in the elderly: new data and retrospective reassessments. Am J Clin Nutr 60: 167-175.

21. Carter ND, Kannus P, Khan KM (2001). Exercise in prevention of falls in older people. A systematic literature review examining the rationale and evidence. Sports Med 31: 427-438.

22. Castaneda C (2003). Diabetes control with physical activity and exercise. Nutr Clin Care 6: 89-96.

23. Castaneda C, Layne JE, Munoz-Orians L, Gordon PL, Walsmith J, Foldvari M, Roubenoff R, Tucker KL, Nelson ME (2002). A randomised controlled trial of resistance exercise training to improve glycemic control in older adults with type 2 diabetes. Diabetes Care 25:L 2335-2341.

24. Chandler JM, Duncan PW, Kochersberg G, Studenski S (1998). Is lower extremity strength gain associated with improvement in physical performance and disability in frail, community-dwelling elders? Arch Phys Med Rehabil 79: 24-30.

25. Chandler JM, Hadley EC (1996). Exercise to improve physiologic and functional performance in old age. Clin Geriatr Med 12: 761-784

26. Charette S, McEvoy L, Pyka G, Snow-Harter C, Guido D, Wiswell R, Marcus R (1991). Muscle hypertrophy response to resistance training in older women. J Appl Physiol 70: 1912-1916.

27. Connelly DM, Vandervoort AA (1995). Improvement in knee extensor strength of institutionalized elderly women after exercise with ankle weights. Physiother Can 47: 15-23.

28. Connelly DM, Vandervoort AA (1997). Effects of detraining on knee extensor strength and functional mobility in a group of elderly women. J Orthop Sports Phys Ther 26: 340-346.

29. Daley MJ, Spinks WL (2000). Exercise, mobility and aging. Sports Med 29: 1-12.

30. Doherty TJ, Vandervoort AA, Brown WF (1993). Effects of ageing on the motor unit: a brief review. Can J Appl Physiol 18: 331-358.

31. Edelberg HK (2001). Falls and function. How to prevent falls and injuries in patients with impaired mobility. Geriatrics 56: 41-45.

32. Eriksson J, Tuominen J, Valle T, Sundberg S, Sovijarvi A, Lindholm H, Tuomilehto J, Koivisto V (1998). Aerobic endurance exercise or circuit-type resistance training for individuals with impaired glucose tolerance? Horm Metab Res 30: 37-41.

33. Evans WJ (1999). Exercise training guidelines for the elderly. Med Sci Sports Exerc 31: 12-17.

34. Faigenbaum AD, Skrinar GS, Cesare WF, Kraemer WJ, Thomas HE (1990). Physiologic and symptomatic responses of cardiac patients to resistance exercise. Arch Phys Med Rehabil 71: 395-398.

35. Feigenbaum MS, Pollock ML (1997). Strength training: rationale for current guidelines for adult fitness programs. Physician Sportsmed 25: 44-64.

36. Ferrandez AM, Pailhous J, Durup M (1990). Slowness in elderly gait. Exp Aging Res 16: 79-89.

37. Fiatarone MA, Marks EC, Ryan ND, Meredith CN, Lipsitz LA, Evans WJ (1990). High intensity strength training in 
nonagenarians. Effects on skeletal muscle. JAMA. 263: 3029-3034.

38. Fiatarone MA, O’Neill EF, Ryan ND, Clements KM, Solares GR, Nelson ME, Roberts SB, Kehayias JJ, Lipsitz LA, Evans WJ (1994). Exercise training and supplementation for physical frailty in very elderly people. $N \mathrm{Engl} \mathrm{J} \mathrm{Med}$ 330: 1769-1775.

39. Fleck SJ, Falkel J, Harman E, Kraemer WJ, Frykman P, Maresh CM, Goetz KL, Campbell D, Rosenstein M, Rosenstein R (1989). Cardiovascular responses during resistance exercise. Med Sci Sports Exerc 21: S114.

40. Fleck SJ, Kraemer WJ (1997). Designing Resistance Training Programs, $2^{\text {nd }}$ Ed. Champaign, Illinois: Human Kinetics.

41. Frontera WR, Hughes VA, Lutz KJ, Evans WJ (1991). A cross-sectional study of muscle strength and mass in 45to 78-yr-old men and women. J App. Physiol 71: 644-650.

42. Frontera WR, Meredith CN, O'Reilly KP, Evans WJ (1990). Strength training and determinants of $\mathrm{VO}_{2} \mathrm{max}$ in older men. J App Physiol 68: 329-333.

43. Frontera WR, Meredith CN, O'Reilly KP, Knuttgen HG, Evans WJ (1988). Strength conditioning in older men: skeletal muscle hypertrophy and improved function. $J$ Appl Physiol 64: 1038-1044.

44. Girouard C, Hurley B (1995). Strength training inhibits gains in shoulder adduction from flexibility training. Med Sci Sports Exerc 27: 1444-1449.

45. Graves JE, Pollock ML, Jones AE, Colvin AB, Leggett SH (1989). Specificity of limited range of motion variable resistance training. Med Sci Sports Med 21: 84-89.

46. Grimby G, Aniansson A, Hedberg M, Henning G-B, Grangard U, Kvist H (1992). Training can improve muscle strength and endurance in 78- to 84-yr-old men. J Appl Physiol 73: 2517-2523.

47. Hagberg J (1990). Exercise Fitness and Hypertension. In Exercise, Fitness and Health: A Consensus of Current Knowledge. Champaign, Illinois: Human Kinetics Publishers, 455-466.

48. Hagerman F, Walsh S, Staron R, Hikida RS, Gilders RM, Murray TF, Toma K, Ragg KE (2000). Effects of high-intensity resistance training on untrained older men. I. Strength, cardiovascular, and metabolic responses. J Geronto. 55A: B336-B346.

49. Hakkinen $\mathrm{K}$, Alen $\mathrm{M}$, Kallinen M, Izquierdo M, Jokelainen K, Lassila H, Malkia E, Kraemer WJ, Newton RU (1998). Muscle CSA, force production, and activation of leg extensors during isometric and dynamic actions in middle-aged and elderly men and women. JAPA 6: 232-247.

50. Hakkinen K, Kraemer WJ, Kallinen M, Linnamo V, Pastinen UM, Newton RU (1996). Bilateral and unilateral neuromuscular function and muscle cross-sectional area in middle-aged and elderly men and women. J Gerontol 51: B21-B29.

51. Hill SR, Piper TJ (2000). Master builders: senior strength training. Natl Strength Cond Assoc J 22: 49-56.

52. Hughes VA, Frontera WR, Wood M, Evans WJ, Dallal GE, Roubenoff R, Fiatarone Singh MA (2001). Longitudinal muscle strength changes in older adults: influence of muscle mass, physical activity and health. J Gerontol 56A: B206-B217.

53. Hurley BF, Hagberg JM (1998). Optimizing health in older persons: aerobic or strength training? In Exercise and Sport Science Reviews. American College of Sports Medicine Series 26: 61-89.
54. Hurley BF, Roth S.M. (2000). Strength training in the elderly. Effects on risk factors for age-related diseases. Sports Med 30: 249-268.

55. Hyatt RH, Whitelaw MN, Bhat A, Scott S, Maxwell JD (1990). Association of muscle strength with functional status of elderly people. Age Aging 19: 330-336.

56. INE (1997). X a XIII Recenseamento Geral da População e estimativas da população residente para 1997 (n²6). Lisboa: Instituto Nacional de Estatística.

57. Izquierdo M, Hakkinen K, Ibanez J, Garrues M, Antón A, Zúniga A, Larrión J, Gorostiaga EM (2001). Effects of strength training on muscle power and serum hormones in middle-aged and older men. J Appl Physiol 100: 14971507.

58. Judge JO, Qunpuu S, Davies III RB (1996). Effects of age on the biomechanics and physiology of gait. Clin Geriatr Med 12: 659-678.

59. Katzel L, Bleecker E, Colman E, Rogus E, Sorkin J, Goldberg A (1995). Effects of weight loss vs. aerobic exercise training on risk factors for coronary disease in healthy, obese, middle-aged and older men. JAMA. 274: 1915-1920.

60. Kell RT, Bell G, Quinney A (2001). Musculoskeletal fitness, health outcomes and quality of life. Sports Med 31: 863-873.

61. Kerr D, Ackland T, Maslen B, Morton A, Prince R (2001). Resistance training over 2 years increases bone mass in calcium-replete postmenopausal women. J Bone Miner Res 16: 175-181.

62. King AC, Taylor CB, Haskell WL (1993). Effects of differing intensities and formats of 12 months of exercise training on psychological outcomes in older adults. Heath Psychol 12:292-300.

63. Kirwan J, Kohrt J, Wojta D, Holloszy J (1993). Endurance exercise training reduces glucose-stimulated insulin levels in 60-to 70-year-old men and women. J Gerontol 48: M84-M100

64. Klitgaard H, Mantono M, Shiaffino S, Ausoni S, Gorza L, Laurent-Winter C, Schnohr P, Saltin B (1990a). Function, morphology and protein expression of ageing skeletal muscle: a cross-sectional study of elderly men with different training backgrounds. Acta Physiol Scand 140: 41-44.

65. Klitgaard H, Zhou M, Shiaffino S, Betto R, Salviati G, Saltin B (1990b). Ageing alters the myosin heavy chain composition of single fibres human skeletal muscle. Acta Physiol Scand 140: 55-62.

66. Kohl HW, LaPorte RE, Blair SN (1988). Physical activity and cancer: an epidemiological perspective. Sports Med 6: 222-237.

67. Kwon S, Oldaker S, Schrager M, Talbot LA, Fozard JL, Metter EJ (2001). Relationship between muscle strength and the time taken to complete a standardized walk-turnwalk test. J Gerontol 56A: B398-B404.

68. Laidlaw DH, Kornatz KW, Reen AD, Suzuki SH, Engra MR (1999). Strength training improves the steadiness of slow lengthening contractions performed by old adults. J Appl Physiol 87: 1786-1795.

69. Lakka TA, Venalainen JM, Rauramaa R, Salonen R, Tuomilehto J, Salonen JT (1994). Relation of leisure-time physical activity and cardiorespiratory fitness to the risk of acute myocardial infarction in men. $N$ Engl J Med 330: 1549-1554.

70. Larsson L, Grimby G, Karlsson J (1979). Muscle strength and speed of movement in relation to age and muscle 
morphology. J Appl Physiol 46: 451-456.

71. Lemmer JT, Hurlbut DE, Martel GF, Tracy BL, Ivey FM, Metter EJ, Fozard, JL, Fleg JL, Hurley BF (2000). Age and gender responses to strength training and detraining. Med Sci Sports Exerc 32: 1505-1512.

72. Lexell J, Downham DY, Larsson Y, Bruhn E, Morsing B (1995). Heavy-resistance training for Scandinavian men and women over seventy: short-and long-term effects on arm and leg muscles. Scand J Med Sci Sports 5: 329-341.

73. Lexell J, Taylor C, Sjostrom M (1988). What is the cause of the ageing atrophy? Total number, size and proportion of different fiber types studies in whole vastus lateralis muscle from 15- to 83-year-old men. J Neurol Sci 84: 275-294.

74. Lipsitz LA, Jonsson PV, Kelley MM, Koestner JS (1991). Causes and correlates of recurrent falls in ambulatory frail elderly. J Gerontol 46: M114-M122.

75. Lynch NA, Metter EJ, Lindle RS, Fozard JL, Tobin JD, Roy TA, Fleg JL, Hurley BF (1999). Muscle quality I. Age-associated diferences between arm and leg muscle groups. $J$ Appl Physiol 86: 188-194.

76. Maddalozzo J, Venkatesan TK, Gupta P (2000). High intensity resistance training: effects on bone in older men and women. Calcify Tissue Int 66: 399-404

77. Marcinik E, Potts J, Schlabach G, Will S, Dawson P, Hurley BF (1991). Effects of strength training on lactate threshold and endurance performance. Med Sci Sports Exerc 23: 739-743.

78. Massey B, Chauder N (1956). Effects of systematic, heavy resistive exercise on range of joint movement in young male adults. Res Q Exerc Sport 27: 41-51.

79. Mazzeo RS, Tanaka H (2001). Exercise prescription for the elderly. Current recommendations. Sports Med 31: 809-818.

80. McDonagh MJN, Davies CTM (1984). Adaptive response of mammalian skeletal muscle to exercise with high loads. Eur J Appl Physiol 52: 139-155.

81. Merssier JP, Dill M (1985). Alterations in strength and maximal oxygen uptake consequent to Nautilus circuit weight training. Res Q Exerc Sport 56: 345-351.

82. Miller JP, Pratley RE, Goldberg AP, Gordon P, Rubin M, Treuth MS, Ryan AS, Hurley BF (1994). Strength training increases insulin action in healthy 50-to 65-yr-old men. $J$ Appl Physiol 77: 1122-1127.

83. Nelson ME, Fiatarone MA, Morgani CM, Trice I, Greenberg RA, Evans WJ (1994). Effects of high-intensity strength training on multiple risk factors for osteoporotic fractures. JAMA. 272: 11009-1914.

84. Overend TJ, Versteegh TH, Thompson E, Birmingham TB, Vandervoort AA (2000). Cardiovascular stress associated with concentric and eccentric isokinetic exercise in young and older adults. J Gerontol 55A: B177-B182.

85. Parker N, Hunter G, Treuth M (1996). Effects of strength training on cardiovascular responses during a submaximal walk and a weight-loaded walking test in older females. $J$ Card Rehab 16: 56-62.

86. Pollock ML, Wilmore JH (1990). Exercise in Health and Disease: Evaluation and Prescription for Prevention and Rehabilitation. W. B. Sauders (Ed.), $2^{\text {nd }}$ Ed. Philadelphia, 91160.

87. Porter MM, Vandervoort AA (1995). High-intensity strength training for the older adult - a review. Top Geriatr Rehabil 10: 61-74.

88. Porter MM, Vandervoort AA, Lexell J (1995). Aging of human muscle: structure, function and adaptability. Scand $J$
Med Sci Sports 5: 129-142.

89. Pratley R, Nicklas B, Rubin M, Miller J, Smith A, Smith M, Hurley B, Goldberg A (1994). Strength training increases resting metabolic rate and norepinephrine levels in healthy 50-to 65-yr-old men. J Appl Physiol 76: 133-137.

90. Proctor DN, Sinning WE, Walro JM, Sieck GC, Lemon PW (1995). Oxidative c,apacity of human muscle fiber types: effects of age and training status. J Appl Physiol 78: 2033-2038.

91. Pruitt LA, Taaffe DR, Marcus R (1995). Effects of a oneyear high-intensity versus low-intensity resistance training program on bone mineral density in older women. J Bone Miner Res 10: 1788-1795.

92. Pyka G, Linderberger E, Charette S, Marcus R (1994). Muscle strength and fiber adaptations to a year-long resistance training program in elderly men and women. $J$ Gerontol 49: M22-M27.

93. Quetelet LAJ (1835). Sur l'homme et le développement de ses facultés. In L. Hauman and Cie, Vol. 2. Paris : Bachelier, Imprimeur-Libraire, 63-77.

94. Rice CL, Cunningham DA, Paterson DH, Dickinson JR (1993). Strength training alters contractile properties of the triceps brachii in men aged 65-78 years. Eur J Appl Physiol 66: 275-280.

95. Ringsberg KA, Gardsell P, Johnell O, Jonsson BB, Obrant KJ, Sernbo I (1998). Balance and gait performance in an urban and a rural population. J Am Geriatr Soc 46: 65-70.

96. Ringsberg KA, Gerdhem P, Johansson J, Obrant KJ (1999). Is there a relationship between balance, gait performance and muscular strength in 75-year-old women? Age Ageing 28: 289-293.

97. Robbins AS, Rubenstein LZ, Josephson KR, Schulman BL, Osterweil D, Fine G (1989). Predictors of falls among elderly people. Arch Int Med 149: 1628-1633.

98. Rogers MA, Evans WJ (1993). Changes in skeletal muscle with aging: effects of exercise training. In Exercise and Sport Science Reviews. American College of Sports Medicine Series 21: 65-102.

99. Roltsch MH, Mendez T, Wilund KR, Hagberg JM (2001). Acute resistive exercise does not affect ambulatory blood pressure in young men and women. Med Sci Sports Exerc 33: 881-886.

100. Roman WJ, Fleckenstein J, Stray-Gundersen J, Always SE, Peshock R, Gonyea WJ (1993). Adaptations in the elbow flexors of elderly males after heavy-resistance training. $J$ Appl Physiol 74: 750-754.

101. Rook KM, Phillips SK, Bruce SA, Woledge RC (1992). The effects of ageing on muscle strength in men and women. $J$ Physiol (London) 452: 25P.

102. Roos MR, Rice CL, Vandervoort AA (1997). Age-related changes in motor unit function. Muscle Nerve 20: 679-6100.

103. Rosa MJ (1999). Reformados e Tempos Livres: resultados do inquérito à população activa e reformada sobre actividades de lazer. Edições Colibri/Inatel.

104. Sagiv M, Hanson P, Besozzi M, Nagle F, Zager L (1985) Left ventricular response to upright isometric handgrip and deadlift in men with coronary artery disease. Am J Cardiol 55: 1298-1302.

105. Schultz AB (1992). Mobility impairment in the elderly: challenges for biomechanics research. J Biomech 25: 519528 .

106. Shaw CE, McCully KK, Posner JD (1995). Injuries during the one repetition maximum assessment in the elderly. $J$ 
Cardiopulm Rehabil 15: 283-287.

107. Shephard RJ. (1990a). Exercise for the frail elderly. Sports Training Med Rehabil 1: 263-277.

108. Shephard RJ (1990b). The scientific basis of exercise prescribing for the very old. Physician Sports Med 11: 91-101.

109. Singh NA, Clements KM, Fiatarone MA (1997). A randomized controlled trial of progressive resistance training in depressed elders. J Gerontol A Biol Sci Med Sci 52: M27-M35.

110. Sipila S, Multanen J, Kallinen M, Era P, Suominen H (1996). Effects of strength and endurance training on isometric muscle strength and walking speed in elderly women. Acta Physiol Scan 156: 457-464.

111. Smolander J, Aminoff I, Khoronen I, Tervo M, Shen N, Korhoren O, Louhevaara V (1998). Heart rate and blood pressure responses to isometric exercise in young and older men. Eur J Appl Physiol 77: 439-444.

112. Spirduso WW (1995). Physical Dimensions of Aging. Champaign, Illinois: Human Kinetics.

113. Stone M, Fleck S, Triplett N, Kramer W (1991). Health and performance related potential of resistance training. Sports Med 11: 210-213.

114. Taaffe DR, Marcus R (1997). Dynamic muscle strength alterations to detraining and retraining in elderly men. Clin Physiol 17: 311-324.

115. Taaffe DR, Pruitt L, Pyka G, Guido D, Marcus R (1996). Comparative effects of high and low-intensity resistance training on muscle strength, fiber area, and tissue composition in elderly women. Clin Physiol 16: 381-392.

116. Taylor AW, Noble EG, Cunningham DA, Paterson DH, Rechnitzer P (1992). Ageing, skeletal muscle contractile properties and enzyme activities with exercise In Y. Sato; J Poortmans; I. Hashimoto; Y. Oshida (Eds.) Integration of Medical and Sport Sciences. Medicine in Sport Sciences. $37^{\text {th }}$ ed. Basel: Karger, 109-125.

117. Thompson LV, Brown M (1999). Age-related changes in contractile properties of single skeletal fibers from the soleus muscle. J Appl Physiol 86: 881-886.

118. Trappe S, Williamson D, Godard M, Porter D, Rowden G, Costill D (2000). Effect of resistance training on single muscle fiber contractile function in older men. J Appl Physiol 89: 143-152.

119. Urbanchek MG, Picken EB, Kalliainen LK, Kuzon Jr. WM (2001). Specific force deficit in skeletal muscles of old rats is partially explained by the existence of denervated muscle fibers. J Gerontol 56A: B191-B197.

120. Vandervoort AA, Chesworth BM, Cunningham DA, Rechnitzer PA, Paterson DH, Kovall JJ (1992). Age and sex effects on mobility of the human ankle. J Gerontol 47: M17-M21.

121. Winett RA, Carpinelli RN (2001). Potential health-related benefits of resistance training. Prev Med 33: 503-513.

122. Yue GH, Raganathan VK, Siemionow V, Liu JZ, Sahgal V (1999). Older adults exhibit a reduced ability to fully activate their biceps brachii muscle. J Gerontol 54A: M249M253. 